\title{
Guidance for management of cancer surgery during the COVID-19 pandemic
}

\author{
Christian Finley, MD, MPH \\ Anubha Prashad, MBA \\ Natasha Camuso, MSc \\ Corinne Daly, MSc \\ Armen Aprikian, MD \\ Chad G. Ball, MD, MSc \\ James Bentley, MBChB \\ Dhany Charest, MD \\ Paola Fata, MD \\ Lucy Helyer, MD, MSc \\ Daniel O'Connell, MD \\ Husein Moloo, MD, MSc \\ Andrew Seely, MD, PhD \\ Joel Werier, MD \\ Toni Zhong, MD, MHS \\ Craig C. Earle, MD, MSc

\section{Correspondence to:}

\section{Finley} \\ Cancer Control \\ Canadian Partnership Against Cancer \\ 145 King Street West \\ Toronto ON M5H 1J8 \\ Christian.Finley@ \\ partnershipagainstcancer.ca
}

DOI: $10.1503 /$ cjs. 005620

\section{SUMMARY}

During the coronavirus disease 2019 (COVID-19) pandemic, delaying lifesaving cancer surgeries must be done with extreme caution and thoughtfulness. Modelling indicates that delays in high-risk cancer surgeries beyond 6 weeks could affect long-term outcomes for thousands of Canadians. Consequently, it is possible that postponing cancer surgery without consideration of its implications could cost more lives than can be saved by diverting all surgical resources to COVID-19. This article provides general guidance on supporting curative surgical treatment where appropriate and with available resources.

D uring the coronavirus disease 2019 (COVID-19) pandemic, the number of cases and deaths may become overwhelming, causing health care leadership and practitioners to face ethical and practical challenges. Several jurisdictions have already started reprioritizing surgical procedures in order to free up health care resources, including ventilators and intensive care unit (ICU) beds, to manage potential COVID-19 cases.

Delaying lifesaving cancer surgeries must be done with extreme caution and thoughtfulness, as delays can have a major impact on long-term survival, patient morbidity and the efficient use of surgical human resources. Postponed cancer surgery can also lead to conditions, such as bowel obstruction or spinal cord impingement, requiring emergent surgeries that otherwise could have been elective. Modelling indicates that delays in high-risk cancer surgeries beyond 6 weeks could affect long-term outcomes for thousands of Canadians. Consequently, it is possible that postponing cancer surgery, if done without consideration of its implications, could cost more lives than can be saved by diverting all surgical resources to COVID-19.

This article provides general guidance on supporting curative surgical treatment where appropriate and with available resources. It reflects the expertise and advice of the executive leadership of the Canadian Network of Surgical Associations for Cancer Care, the Canadian Association of Provincial Cancer Agencies, the Canadian Partnership Against Cancer and clinical cancer experts across the country. Recommendations from Canadian and international jurisdictions have been used to inform this guideline.

\section{GUIDANCE ON COMPETING NEEDS AND PRIORITIZATION CRITERIA}

Within Canada, many provinces have developed "cancer patient priority classification" systems to assist cancer programs in the management of cancer patients (Box 1). This must be contextualized within the current load on the system and anticipated trajectory, not only of a COVID-19 caseload, but also a backlogged cancer caseload, both of which are populations with critical lifesaving surgical needs. 
Contemporaneous decisions will be needed that are data driven where possible and that consider today's and tomorrow's needs with at least a medium timeframe; we recommend a 6-month view of management in the Canadian health care system. The following are common guiding principles that we believe should form the basis of a panCanadian approach to this problem.

\section{Cancer surgery should be designated "essential"}

Most cancer surgeries are of high priority and curative. Cancer surgery should continue to be designated as "essential" and should be among the last types of surgery to be delayed. ${ }^{1}$

\section{Transfer care of cancer patients to less overwhelmed institutions as a first-line strategy}

Once a hospital reaches a critical mass of patients receiving mechanical ventilation, nonemergency surgeries will not be able to be carried out. Under these circumstances, we recommend jurisdictional, then regional coordination among health authorities, institutions and surgeons to transfer care of cancer patients to less overwhelmed institutions as a first-line strategy, rather than delaying cancer surgery. ${ }^{2}$

\section{High-level guidance around prioritization is needed if delaying cancer surgery becomes necessary}

If delaying cancer surgery becomes necessary, we recommend high-level pan-Canadian and jurisdictional guidance around prioritization, with explicit prioritization tiers, to bring transparency and consistency to the approach, recognizing that ultimate decision making will be need to be done locally. ${ }^{1}$ Examples of resources for establishing evidence- and risk-based prioritization are shown in Box 1. Whichever tool is used, it will need to recognize that some cancer patients require more urgent care, while others can safely wait a longer period of time.

- Ideally, the prioritization tool used by a jurisdiction should be linked to the changing level of an institution's surgical resources to guide decision making regarding cancer surgery cases to be performed/ delayed.

- Any triage of patients must be done equitably. Clinical triage for major surgery should be guided by ethical principles. Relevant ethical principles are utility, proportionality and fairness. ${ }^{3}$

\section{There must be concrete plans in place to carry out delayed surgeries within a reasonable period of time}

Multidisciplinary care should be leveraged on a case-bycase basis to enable safer delays for some cancer surger-
Box 1: Resources providing directives around management of surgical oncology during the COVID-19 pandemic

- Cancer Care Ontario. Pandemic planning clinical guideline for patients with cancer. https://www.accc-cancer.org/docs/documents/cancerprogram-fundamentals/oh-cco-pandemic-planning-clinical-guideline_ final_2020-03-10.pdf

- Ontario Health. Clinical triage protocol for major surge in COVID pandemic: https://emergencymedicinecases.com/wp-content/ uploads/2020/04/Clinical-Triage-Protocol-for-Major-Surge-in-COVID -Pandemic-March-28-2020.pdf

- Ministère de la Santé et des Services sociaux. COVID-19 pour les professionnels: https://www.msss.gouv.qc.ca/professionnels/

- Cancer Control Alberta. COVID-19 Planning Clinical Guidance for Patients with Cancer.

- Nova Scotia Health Authority. NSHA Perioperative and Interventional Radiology Services During COVID-19 Pandemic: https://www.cdha. nshealth.ca/system/files/sites/documents/perioperative-and-ir-services -during-covid3204pm.pdf

- Anderson H, Tyldesley S, Pansegrau G, Chi K. (British Columbia, March 20 2020). Criteria for Clinical Prioritization During the COVID-19 Pandemic.

- Cancer Care Manitoba: Action Cancer Manitoba. (March 15, 2020). Clinical Management Framework - Systemic Therapy and Radiotherapy for Cancer Patients and Those with Serious Blood Disorders in the Context of the COVID-19 Pandemic.

- Australian Government - Cancer Australia. Information about cancer and COVID-19 - Surgery. https://canceraustralia.gov.au/affected-cancer/ information-about-cancer-and-covid-19/health-professionals/surgery

- National Comprehensive Cancer Network: https://jnccn.org/view/ journals/jnccn/18/4/article-p366.xml?rskey=vYDEqI\&result=1

- American College of Surgeons. COVID 19: elective case triage guidelines for surgical care: https://www.facs.org/-/media/files/covid19/ guidance_for_triage_of_nonemergent_surgical_procedures.ashx

- National Health Service. Clinical guide for the management of noncoronavirus patients requiring acute treatment: Cancer. https://www. england.nhs.uk/coronavirus/wp-content/uploads/sites/52/2020/03/ specialty-guide-acute-treatment-cancer-23-march-2020.pdf

- European Cancer Organization. News: statement on COVID-19 from the European Cancer Organisation's board of directors: https://www.ecco -org.eu/Global/News/Latest-News/2020/03/NEWS-Statement-on-COVID -19-from-the-European-Cancer-Organisation-Board-of-Directors

- The Lancet Oncology. The official French guidelines to protect patients with cancer against SARS-CoV-2 infection: https://www.thelancet.com/ journals/lanonc/article/PIIS1470-2045(20)30204-7/fulltext

ies (e.g., when temporizing treatments, such as neoadjuvant chemotherapy and/or radiation, are available). However, it is likely that this pandemic will stretch over many months until herd immunity, an effective treatment and/or a vaccine stems the tide of new cases. There must be concrete plans in place to carry out delayed surgeries within a reasonable period of time, even if the pandemic is not over.

\section{Recovery planning for resumption of surgical services after the pandemic should start now}

Eventually this pandemic will pass, and we will be faced with catching up on a backlog of surgical cancer cases. This means that the health system will need to operate at a surge well past the end of the COVID-19 crisis. Health system planners must account for this surge, as well as what care can continue to be provided during the 
next period of time, in their future projections. Recovery planning for resumption of surgical services after the pandemic should start now and be communicated on a go-forward basis as the health system stabilizes; this planning should include an impact analysis of the projected long-term health care costs and human resource needs caused by delayed cancer treatment.

Affiliations: From the Canadian Partnership Against Cancer (Finley, Prashad, Camuso, Daly, Earle); the Canadian Network of Surgical Associations for Cancer Care (Aprikian, Ball, Bentley, Charest, Fata, Helyer, O'Connell, Moloo, Seely, Werier, Zhong); the Canadian Urological Association and the Division of Urology, Department of Surgery, McGill University, Montreal, Que. (Aprikian); the Canadian Hepato-PancreaticoBiliary Association and the Department of Surgery, University of Calgary, Calgary, Alta. (Ball); the Society for Gynecologic Oncology of Canada and the Department of Obstetrics and Gynaecology, Dalhousie University, Halifax, NS (Bentley); the Canadian Neurosurgical Society and the Department of Neurosurgery, Dalhousie University New Brunswick, Moncton, NB (Charest); the Canadian Association of General Surgeons and the Division of General Surgery, Department of Surgery, McGill University, Montreal, Que. (Fata); the Canadian Society of Surgical Oncology and the Department of General Surgery, Dalhousie University, Halifax, NS (Helyer); the Canadian Association of Head and Neck Surgical Oncology and the Division of Otolaryngology - Head and Neck Surgery, University of Alberta, Edmonton, Alta.(O'Connell); the Canadian Society of Colon and Rectal Surgeons and the Division of General Surgery, Department of Surgery, University of Ottawa, and the Ottawa Hospital Research Institute, Ottawa, Ont. (Moloo); the Canadian Association of Thoracic Surgeons and the Department of Cellular and Molecular Medicine, University of Ottawa, Ottawa, Ont. (Seely); the Canadian Orthopaedic Oncology Society and the Division of Orthopaedic Surgery, Department of Surgery, University of Ottawa, Ottawa, Ont. (Werier); the Canadian Society of Plastic Surgeons and the Department of Surgery, University of Toronto, Toronto, Ont. (Zhong); the Department of Surgery, McMaster University, Hamilton, Ont. (Finley); the Department of Gastrointestinal Oncology, Sunnybrook Health Sciences Centre, Toronto, Ont. (Earle); and the Canadian Fournal of Surgery (Ball).
Competing interests: J. Bentley is a member of the advisory boards of AstraZeneca and NSV Tech, and his institution receives funding from Merk, AstraZeneca and Clovis for trials he is involved in. T. Zhong is supported by a Canandian Institutes of Health Research (CIHR) New Investigator Award (2015-2020) and she is the inaugural Belinda Stronach Chair in Breast Cancer Reconstruction Surgery at the University Health Network (2017-2022). C. Ball is co-editor in chief of CFS; he was not the accepting editor on this manuscript. No other competing interests were declared.

Contributors: All authors contributed substantially to this guidance article. C. Finley, A. Prashad, C. Daly, L. Helyer, D. O'Connell, H. Moloo, J. Werier, T. Zhong and C. Earle conceived the idea for the article. A. Prashad, N. Camuso and C. Ball compiled the current best evidence, which all authors assessed. C. Finley, A. Prashad, N. Camuso, C. Daly, C. Ball, A. Seely, T. Zhong and C. Earle wrote the article, which all authors reviewed and approved for publication.

Acknowledgements: Both the Canadian Partnership Against Cancer and the Canadian Network of Surgical Associations for Cancer Care would like to acknowledge the valuable contributions of the Canadian Association of Provincial Cancer Agencies for their input and guidance in the production of the document.

\section{References}

1. American College of Surgeons. COVID 19: elective case triage guidelines for surgical care. 2020 Mar. 27. Available: https://www.facs.org/-/ media/files/covid19/guidance_for_triage_of_nonemergent_surgical_ procedures.ashx (accessed 2020 Mar. 31).

2. Cancer Care Ontario. Pandemic Planning Clinical Guideline for Patients with Cancer. 2020 Mar. 10. Available: https://www.accc-cancer.org/docs/ documents/cancer-program-fundamentals/oh-cco-pandemic-planning -clinical-guideline_final_2020-03-10.pdf (accessed 2020 Mar. 31).

3. Ontario Health. Clinical Triage Protocol for Major Surge in COVID Pandemic. 2020 Mar. 28. Available: https://emergencymedicinecases.com/ wp-content/uploads/2020/04/Clinical-Triage-Protocol-for-Major-Surge -in-COVID-Pandemic-March-28-2020.pdf (accessed 2020 Mar. 31). 